\title{
Reduced Symmetry of Heterointerfaces and Orientational Pinning of Quantum Hall Stripe Phase
}

\author{
E.E. Takhtamirov ${ }^{\dagger}$ and V.A. Volkov \\ Institute of Radioengineering and Electronics of RAS \\ Mokovaya 11, 101999, Moscow, Russia \\ † E-mail: takhtam@cplire.ru
}

\begin{abstract}
In a 2D electron system in (001) GaAs/AlGaAs upon filling high Landau levels, it was recently observed a new class of collective states, which are recognized now to relate to the spontaneous formation of a charge density wave ("stripe phase"). Here we analyze one of the possible mechanisms of the stripe pinning along the crystallographic direction [110] - the native effective mass anisotropy of $2 \mathrm{D}$ electrons. It is shown that for a symmetric quantum well this anisotropy is a linear function of applied perpendicular-to-plane electric field. So, if it is the anisotropy that defines the direction of the stripes, the critical in-plane magnetic field, when the stripes rotate, will strongly depend on the bias. The proper experiment will answer the question whether the native anisotropy of the effective mass is the mechanism of the pinning.
\end{abstract}

\section{Introduction}

The experiments by Lilly, et al. and Du, et al. [1] revealed a new class of collective states of $2 \mathrm{D}$ electron system in excited Landau levels in the single heterojunction (001) GaAs/AlGaAs. Now it is generally accepted that these states are related to spontaneous formation of the unidirectional charge density wave (quantum Hall stripe phase) predicted and calculated earlier [2]. This point of view was primarily based on the observation of a giant resistance anisotropy in this system. The ratio of resistances along the crystallographic directions [1ㅣ] and [110] reaches the values of $R_{x x} / R_{y y} \sim 5$-3500, depending on the sample geometry, where [110] is the "easy" conductivity direction. 
Moreover, the behavior of all conductivity tensor components qualitatively agrees with the theory [3].

It was shown that the in-plane magnetic field $B_{\|} \sim 1 \mathrm{~T}$ can change the direction of easy conductivity [4]. It was made a conclusion that, at high enough $B_{\|}$, the direction of easy conductivity is perpendicular to the $\mathbf{B}_{\|}$ direction. The theoretical analysis [5] of the influence of $\mathbf{B}_{\|}$carried out in the Hartree-Fock approximation partially explained the results. But among researchers still there is no agreement on the native mechanism that makes the stripes be pinned coherently across the sample along the crystallographic direction [110] at $B_{\|}=0$.

It was supposed by Kroemer [6] that the reduced symmetry $C_{2 v}$ of the heterojunction composed of III-V semiconductors might be the origin of the orientational pinning of the quantum Hall stripe phase. Adopting this idea we have recently showed [7] that for a typical single-interface structure the effective masses along [110] and [110] directions differ by about $0.1 \%$ ("native" anisotropy). The in-plane magnetic field $\mathbf{B}_{\|}$leads to the "magnetic" anisotropy of the effective mass $\left(\propto B_{\|}^{2}\right)$. The $2 \mathrm{D}$ electron system with the anisotropic mass and isotropic Coulomb interaction is equivalent to that one with the isotropic (cyclotron) mass and anisotropic Coulomb interaction. We supposed that it was the effective anisotropic interaction that pinned the orientation of the stripes and allowed to detect the phase in the magnetotransport experiments. The interplay of the native and magnetic contributions to the effective mass anisotropy leads at $B_{\|} \sim 0.5 \mathrm{~T}$ to the isotropic electron spectrum if $\mathbf{B}_{\|}$along [110]. As a result, the direction of the stripes becomes chaotic and the conductivity turns isotropic. Further increase of $B_{\|}$makes magnetic anisotropy dominate and the stripes align along [11̄0] (perpendicular to $\mathbf{B}_{\|}$for the one-subband case, and parallel to $\mathbf{B}_{\|}$for the two-subband case). The results seem to be in quantitative agreement with the experimental findings.

Here we will consider the orientational pinning of the stripe phase in a square quantum well. The goal of the paper is to find a procedure that will unambiguously give the answer on the question whether the native anisotropy of the effective mass is the mechanism of pinning of the quantum Hall stripe phase.

\section{Symmetric quantum well in perpendicular electric field}

For a symmetric quantum well in the perpendicular-to-plane magnetic 
field the crystallographic directions [110] and [1ํㅣ are mutually equivalent. The system has $D_{2 d}$ symmetry, and the conductivity should be anisotropic only as an in-plane magnetic field is applied. This is in agreement with the recent data by Pan, et al. [8].

We analyse the single-particle Hamiltonian for electron in a square quantum well with heterointerfaces at $z=0$ and $z=L$. Let us assign $x \|[1 \overline{1} 0]$, $y \|[110]$ and $z \|[001]$, and introduce magnetic field $\mathbf{B}$ in the vector-potential gauge $\mathbf{A}=\left(B_{y} z,-B_{x} z+B_{z} x, 0\right)$. Following Refs. [7,9] we may obtain the orbital part of the 3D Hamiltonian:

$$
\begin{aligned}
H_{3 D}= & \frac{p_{z}^{2}}{2 m^{*}}+V(z)+\frac{1}{2}\left(\frac{1}{m^{*}}-\alpha_{1} \delta(z)+\alpha_{2} \delta(z-L)\right)\left(p_{x}+\frac{e}{c} B_{y} z\right)^{2} \\
& +\frac{1}{2}\left(\frac{1}{m^{*}}+\alpha_{1} \delta(z)-\alpha_{2} \delta(z-L)\right)\left(p_{y}-\frac{e}{c} B_{x} z+\frac{e}{c} B_{z} x\right)^{2} .
\end{aligned}
$$

Here, $\mathbf{p}$ is the momentum operator, $m^{*}$ is the band edge effective mass, $V(z)$ is the effective potential of the conduction band edge, $e$ is the elementary charge, $c$ is the speed of light, $\delta(z)$ is the Dirac $\delta$-function. If the quantum well is true symmetric, symmetric is the potential $V(z)$, and the interface parameters $\alpha_{1}$ and $\alpha_{2}$, see Ref. [7], are equal, $\alpha_{1}=\alpha_{2}=\alpha$. Generally these parameters may differ as different are the growth conditions for the direct, AlGaAs/GaAs, and inverted, GaAs/AlGaAs, heterointerfaces.

For a 2D layer of finite thickness the in-plane component of magnetic field can be treated perturbatively. To the second order in $B_{\|}$this procedure brings about a diamagnetic shift of $2 \mathrm{D}$ subband energies, shift of the center of the Landau orbit in 2D quasi-momentum space, and an increase (for the lowest subband) in the effective mass in the direction perpendicular to $\mathbf{B}_{\|}$. The terms proportional to $\alpha_{i}(i=1,2)$ in Eq. (11) also can be treated perturbatively. For simplicity, we assume that $\mathbf{B}_{\|}$is parallel to either [1]0] or [110], so that $B_{x} B_{y}=0$. Collecting all terms second-order in $B_{\|}$and first-order in $\alpha_{i}$, and one obtains the following expression for 2D Hamiltonian of the ground subband [7]:

$$
\begin{aligned}
H_{2 D}^{1} & =E_{1}+\frac{e^{2}}{2 m^{*} c^{2}}\left(B_{x}^{2}+B_{y}^{2}\right)\left(\left\langle 1\left|z^{2}\right| 1\right\rangle-\langle 1|z| 1\rangle^{2}\right) \\
& +\frac{1}{2 m^{*}}\left(1-\frac{\Delta_{\text {nat }}}{2}-\frac{B_{y}^{2}}{B_{\|}^{2}} \Delta_{\mathrm{B}}\right)\left(p_{x}+\frac{e}{c} B_{y}\langle 1|z| 1\rangle\right)^{2}
\end{aligned}
$$




$$
+\frac{1}{2 m^{*}}\left(1+\frac{\Delta_{\text {nat }}}{2}-\frac{B_{x}^{2}}{B_{\|}^{2}} \Delta_{\mathrm{B}}\right)\left(p_{y}+\frac{e}{c} B_{z} x-\frac{e}{c} B_{x}\langle 1|z| 1\rangle\right)^{2} .
$$

Here $|m\rangle=\Phi_{m}(z)$ is $z$-motion envelope function of $m$ th subband, it is taken to be real. The parameters of the natural anisotropy of effective mass and its anisotropy induced by the magnetic field are

$\Delta_{\text {nat }}=2 m^{*}\left\langle 1\left|\alpha_{1} \delta(z)-\alpha_{2} \delta(z-L)\right| 1\right\rangle, \quad \Delta_{\mathrm{B}}=\frac{2 e^{2} B_{\|}^{2}}{m^{*} c^{2}} \sum_{m}^{\prime} \frac{\langle 1|z| m\rangle^{2}}{E_{m}-E_{1}}$

where $E_{m}$ is the energy of the bottom of the $m$ th subband at $B=0$.

For a symmetric quantum well $\Delta_{\text {nat }}=0$. Nevertheless, when the perpendicular-to-plane electric field $\mathbf{F}$ is applied, the system gets $C_{2 v}$ symmetry and should behave like a single-interface one. That means $\Delta_{\text {nat }} \neq 0$ being a linear function of $F$. It is the consequence of the linear Stark effect, which arise as the Hamiltonian (1) does not possess the symmetry $H_{3 D}(z)=H_{3 D}(L-z)$. Consider the energy $e F z$ as a perturbation; $\Phi_{m}$ will be unperturbed wave functions. Then

$$
\Delta_{\text {nat }}=4 m^{*} \alpha e F \sum_{m}^{\prime} \frac{\langle 1|z| m\rangle}{E_{m}-E_{1}}\left(\Phi_{1}(0) \Phi_{m}(0)-\Phi_{1}(L) \Phi_{m}(L)\right) .
$$

Generally, for any structure $\Delta_{\text {nat }}=C+D \cdot F$, where $C$ and $D$ are some parameters. For a symmetric quantum well $C=0$.

Let us analyze some recent experimental results by Cooper, et al. [10]. In particular, for a formally symmetric quantum well (001) GaAs $/ \mathrm{Al}_{0.24} \mathrm{Ga}_{0.76} \mathrm{As}$ they found that the resistance was anisotropic even without the in-plane magnetic fieled. This result is in contrast with data by Pan, et al. [8]. Two possible explanations of the contradictory may be given. The first one relates to the difference of the interface parameter $\alpha$ for the direct and inverted heterointerfaces in the sample of Ref. [10], albeit the quantum well was intended to be grown symmetric. Another explanation is based on existence of unintentional perpendicular-to-plane electric field, which pushes the electron wave function onto one of the heterointerfaces and induces the effective mass anisotropy (4).

We present some results of the selfconsistent Shrödinger-Poisson computing and comparison with the experiment, Ref. [10]. For the heterojunction $\mathrm{GaAs} / \mathrm{Al}_{0.3} \mathrm{Ga}_{0.7} \mathrm{As}$ from comparison with the experiments we obtained the value $\alpha=1.1 \cdot 10^{20} \mathrm{~cm} / \mathrm{g}$ [7]. The linear interpolation for the symmetric 
quantum well GaAs with bariers $\mathrm{Al}_{0.24} \mathrm{Ga}_{0.76}$ As gives $\alpha_{1}=0.88 \cdot 10^{20} \mathrm{~cm} / \mathrm{g}$. Assume the heterointerfaces in the sample of Ref. [10] not equivalent, and no unintentional perpendicular-to-plane electric field presents. Then from the condition $\Delta_{\text {nat }}=\Delta_{\mathrm{B}}$ at critical magnetic field $B_{\|}=0.24 \mathrm{~T}$, when the resistance becomes isotropic, we get $\alpha_{2}=-3.1 \cdot 10^{20} \mathrm{~cm} / \mathrm{g}$. This value looks strange, but no one can exclude such a case. Suppose now the quantum well microscopically symmetric, $\alpha_{1}=\alpha_{2}=0.88 \cdot 10^{20} \mathrm{~cm} / \mathrm{g}$. Then the electric field $F=1.5 \cdot 10^{4} \mathrm{~V} / \mathrm{cm}$ is able to induce the effective mass anisotropy that will be cancelled in the magnetic field $B_{\|}=0.24 \mathrm{~T}$. Note, that the sum in (3) rapidly diminishes with decreasing the width of the quantum well (while $L=300 \AA$ in Ref. [10]). If the native anisotropy of the effective mass exists, the critical in-plane magnetic field will be higher for narrower quantum wells.

\section{Conclusion}

We analyzed the possible mechanisms of the quantum-Hall stripe pinning along the crystallographic direction [110] - the native effective mass anisotropy of $2 \mathrm{D}$ electrons. It was shown that for a symmetric quantum well this anisotropy was a linear function of applied perpendicular-to-plane electric field. If it is the anisotropy that defines the direction of the stripes, the critical in-plane magnetic field, when the resistance goes isotropic, will strongly depend on the bias. The proper experiment will answer the question whether the native anisotropy of the effective mass is the mechanism of the pinning.

The work was supported by RFBR (99-02-17592 and 01-02-06476), Federal Programs FTNS and PAS. Besides, E.E.T. was supported in part by the Young Scientists Support Program of RAS (Grant \#43).

\section{References}

[1] M.P. Lilly, et al., Phys. Rev. Lett. 82, 394 (1999); R.R. Du, et al., Solid State Commun. 109, 389 (1999).

[2] M.M. Fogler, et al., Phys. Rev. B 54, 1853 (1996); M.M. Fogler and A.A. Koulakov, ibid 55, 9326 (1997); R. Moessner and J.T. Chalker, ibid 54, 5006 (1996).

[3] G.R. Aizin and V.A. Volkov, Sov. Phys. JETP 60, 844 (1984); ibid 65, 
188 (1987); A.H. MacDonald and M.P.A. Fisher, Phys. Rev. B 61, 5724 (2000); F. von Oppen, et al., Phys. Rev. Lett. 84, 2937 (2000).

[4] W. Pan, et al., Phys. Rev. Lett. 83, 820 (1999); M.P. Lilly, et al., ibid 83, 824 (1999).

[5] T. Jungwirth, et al., Phys. Rev. B 60, 15574 (1999); T.D. Stanescu, et al., Phys. Rev. Lett. 84, 1288 (2000).

[6] H. Kroemer, cond-mat/9901016.

[7] E.E. Takhtamirov and V.A. Volkov, JETP Lett. 71, 422 (2000), condmat/0006226.

[8] W. Pan, et al., Phys. Rev. Lett. 85, 3257 (2000).

[9] E.E. Takhtamirov and V.A. Volkov, JETP 89, 1000 (1999).

[10] K.B. Cooper, et al., cond-mat/0104243. 